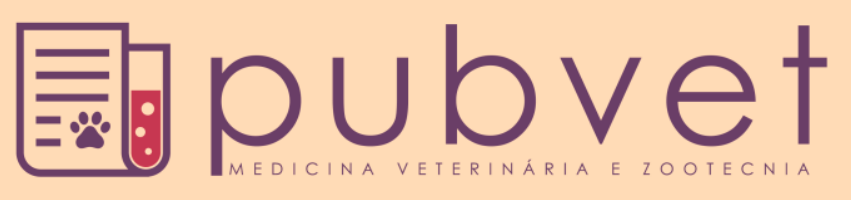

ISSN $1982-1263$

https://doi.org/10.31533/pubvet.v12n8a158.1-6

\title{
Hepatite crônica focal em cão Golden Retriever: Relato de caso
}

\author{
Camila Lúcio Pereira ${ }^{1^{*}} \bullet$, Nathália Fernanda Pereira de $\operatorname{Godoy}^{1} \bullet$, Érika Maria \\ Terra $^{2} \bullet$, Artur Gouveia Rocha ${ }^{3}$
}

${ }^{I}$ Graduanda em Medicina Veterinária pelo Centro Universitário Central Paulista - UNICEP, São Carlos -SP, Brasil

${ }^{2}$ Professora Doutora de Clínica Médica de Pequenos Animais pelo Centro Universitário Central Paulista - UNICEP, São Carlos-SP, Brasil ${ }^{3}$ Professor Doutor de Clínica Cirúrgica de Pequenos Animais pelo Centro Universitário Central Paulista - UNICEP, São Carlos-SP, Brasil

*Autor para correspondência, E-mail: camila_usp@yahoo.com.br

RESUMO. A hepatite crônica canina é uma doença progressiva e irreversível, de etiologia variada, que causa diminuição da regeneração hepática e substituição do parênquima por fibrose, podendo evoluir para cirrose. O objetivo do presente trabalho foi relatar o caso de uma cadela da raça Golden Retriever, com 11 anos e 3 meses de idade, atendida no Hospital Veterinário Universitário-HVU, São Carlos/SP, Brasil. O motivo da consulta foi infecção por Giardia sp, mas, os exames laboratoriais para avaliar as funções biológicas da paciente, resultaram em elevados valores de ALT (alanina aminotransferase) e FA (fosfatase alcalina), além de hipoalbuminemia, indicando lesão hepática severa, porém, sem apresentar outros sinais clínicos comuns em cães hepatopatas (icterícia, ascite, vômito, etc.). Os exames de imagem, ultrassonografia e tomografia computadorizada, registraram hepatomegalia e presença de estrutura amorfa única de contornos irregulares e parênquima heterogêneo. Como tratamento cirúrgico foi eleita a hepatectomia parcial para a retirada do lobo medial direito onde estava a massa. Para o tratamento clínico foram prescritos: Ursacol (ácido ursodesoxicólico), SAM-e (S-Adenosil-Metionina) e vitamina E, diariamente. O histopatológico da amostra resultou em hiperplasia hepatocelular com formação de pontes de fibrina e nódulos regenerativos, indicativos de hepatite crônica, além de ausência de características de malignidade ou agentes infecciosos. A paciente foi acompanhada nos meses seguintes ao tratamento, com acentuada redução dos valores séricos das enzimas hepáticas e manutenção da boa saúde. Conclui-se que o diagnóstico de hepatite crônica, antes do surgimento de sinais clínicos, associado à hepatectomia parcial e ao tratamento clínico melhoram o prognóstico e sobrevida dos pacientes com doença hepática focal.

Palavras chave: fígado, cão, hepatite, hiperplasia hepatocelular

\section{Chronic focal hepatitis in Golden Retriever dog: Case report}

ABSTRACT. Chronic canine hepatitis is a progressive and irreversible disease with a varied etiology that causes decreased liver regeneration and parenchyma replacement due to fibrosis, which may progress to cirrhosis. The objective of the present study was to report the case of a Golden Retriever bitch, 11 years and 3 months old, attended at the University Veterinary Hospital-HVU, São Carlos / SP, Brazil. The reason for the consultation was infection with Giardia sp, but laboratory tests to evaluate the biological functions of the patient resulted in high ALT (alanine aminotransferase) and FA (alkaline phosphatase), in addition to hypoalbuminemia, indicating severe hepatic injury, however, without presenting any other clinical signs common in hepatopathy dogs (jaundice, ascites, vomiting, etc.). Ultrasonography and computed tomography showed hepatomegaly and the presence of a single amorphous structure with irregular contours and heterogeneous parenchyma. As a surgical treatment, partial hepatectomy was chosen for removal of the right medial lobe where the mass was located. Ursacol (ursodeoxycholic acid), SAM-e (S- 
Adenosyl-Methionine) and vitamin E were prescribed for clinical treatment on a daily basis. Histopathology of the sample resulted in hepatocellular hyperplasia with formation of fibrin bridges and regenerative nodules, indicative of chronic hepatitis, in addition to absence of malignancy characteristics or infectious agents. The patient was followed up in the months following the treatment, with a marked reduction in serum liver enzymes and maintenance of good health. It is concluded that the diagnosis of chronic hepatitis, before the appearance of clinical signs, associated with partial hepatectomy and clinical treatment improves the prognosis and survival of patients with focal liver disease.

Keywords: liver, dog, hepatitis, hepatocellular hyperplasia

\section{Hepatitis crónica focal em canino Golden Retriever: Reporte de un caso}

RESUMEN. La hepatitis crónica canina es una enfermedad progresiva e irreversible, de etiología variada, que causa disminución de la regeneración hepática y sustitución del parénquima por fibrosis, pudiendo evolucionar hacia cirrosis. El objetivo del presente trabajo fue relatar el caso de una perra de la raza Golden Retriever, con 11 años y 3 meses de edad, atendida en el Hospital Veterinario Universitario-HVU, São Carlos / SP, Brasil. El motivo de la consulta fue infección por Giardia sp, pero los exámenes de laboratorio para evaluar las funciones biológicas de la paciente, resultaron en elevados valores de ALT (alanina aminotransferasa) y FA (fosfatasa alcalina), además de hipoalbuminemia, indicando lesión hepática severa, pero sin presentar otros signos clínicos comunes en perros hepatopáticos (ictericia, ascitis, vómito, etc.). La ultrasonografía y tomografía computarizada, registraron hepatomegalia y presencia de estructura amorfa única de contornos irregulares y parénquima heterogéneo. Como tratamiento quirúrgico fue elegida la hepatectomía parcial para la retirada del lobo medial derecho donde estaba la masa. Para el tratamiento clínico se prescribieron: Ursacol (ácido ursodesoxicólico), SAM-e (SAdenosil-Metionina) y vitamina E, diariamente. El histopatológico de la muestra resultó en hiperplasia hepatocelular con formación de puentes de fibrina y nódulos regenerativos, indicativos de hepatitis crónica, además de ausencia de características de malignidad o agentes infecciosos. La paciente fue acompañada en los meses siguientes al tratamiento, con acentuada reducción de los valores séricos de las enzimas hepáticas y mantenimiento de la buena salud. Se concluye que el diagnóstico de hepatitis crónica, antes del surgimiento de signos clínicos, asociado a la hepatectomía parcial y al tratamiento clínico, mejoran el pronóstico y la supervivencia de los pacientes con enfermedad hepática focal.

Palabras clave: hígado, perro, hepatitis, hiperplasia hepatocelular

\section{Introdução}

$\mathrm{O}$ fígado tem papel fundamental no equilíbrio de diversos processos biológicos, com estimativa de realizar ao menos 1500 funções bioquímicas essenciais ao organismo, tornando-o sujeito a vários tipos de lesões. Entre as diversas atividades desenvolvidas pelo fígado, podemos destacar o metabolismo de proteínas, carboidratos, lipídeos, vitaminas e minerais, além da desintoxicação do sangue portal, a formação e excreção da bile, a produção de albumina e a síntese de fatores de coagulação (Schwarz, 2012).

A hepatite crônica é considerada um distúrbio secundário a uma das diversas causas possíveis de injúria hepática, em que a arquitetura do fígado é alterada devido fibrose hepática difusa no parênquima lobular, sendo um processo crônico, progressivo e irreversível, mas, comum em animais domésticos (Richter, 2005; Webster, 2008). Na medicina veterinária a etiologia da hepatite é, na maioria das vezes difícil de ser definida, pois, sua ocorrência está associada à uma ampla variedade de causas de lesão hepatocelular, como: adenovírus canino tipo 1 (CAV-1); predisposição racial dos cães Bedlington Terrier, West Highland White Terrier, Doberman Pinscher, Skye Terrier e Cocker Spaniel (Silva et al., 2007); exposição contínua a toxinas (aflatoxicose crônica) ou medicamentos anticonvulsivantes; lesões imunológicas; ou idiopática quando foram excluídas todas as etiologias conhecidas.

Apesar da existência de extensas descrições morfológicas de hepatites crônicas e de uma 
grande variedade de classificações propostas, a maioria dos casos de hepatite crônica é referida como idiopáticas, em que os estudos em hepatologia canina ainda apresentam etiologia limitada, quando comparado às hepatites em humanos (Tostes \& Bandarra, 2004). Assim, este trabalho tem como objetivo relatar a ocorrência de hepatite crônica focal em um cão Golden Retriever.

\section{Relato de caso}

Uma cadela da raça Golden Retriever de 11 anos e 3 meses de idade e pesando $29 \mathrm{~kg}$ foi atendida no Hospital Veterinário Universitário HVU/UNICEP, São Carlos/SP, em janeiro de 2017. A queixa principal era a presença de diarreia aguda e hiporexia. Durante a anamnese os tutores relataram que outros dois animais do domicílio também apresentavam diarreia aguda, o que resultou no diagnóstico de giardíase e foi iniciado o tratamento com fembendazol $500 \mathrm{mg}$ (Fenzol $\mathrm{Pet}^{\circledR}$ ), 3 comprimidos, a cada 24 horas durante 3 dias, com repetição do tratamento após 15 dias. A vacinação do canino estava atualizada (V10 e antirrábica) e, durante o exame físico, todos os parâmetros fisiológicos se mostraram dentro dos valores de normalidade. Contudo, aproveitando a consulta os médicos veterinários solicitaram a realização de exames complementares, hemograma e bioquímico sérico, a saber: creatinina, ureia, ALT (alanina aminotransferase), FA (fosfatase alcalina) e albumina. O hemograma não demonstrou qualquer tipo de alteração hematológica. No entanto, os resultados do bioquímico sérico apresentaram valores elevados para ALT (406 U.I./L) e FA (2640 U.I./L) e hipoalbuminemia $(1,8 \mathrm{~g} / \mathrm{dL})$, já os valores de creatinina e ureia $(0,86 \mathrm{mg} / \mathrm{dL}$ e $41 \mathrm{mg} / \mathrm{dL}$, respectivamente), usados para avaliar a função renal, apresentaram-se dentro dos valores de referência para a espécie. Diante dos elevados valores de ALT e FA foi realizada ultrassonografia abdominal, que registrou hepatomegalia com presença de estrutura amorfa de contornos irregulares e parênquima heterogêneo em região adjacente aos lobos hepáticos mediais, sugestivo de neoplasma hepático focal (Figura 1).

Mediante os resultados dos exames complementares, trinta dias depois, o animal foi encaminhado para a realização de tomografia torácica e abdominal (técnica $80 \mathrm{mAs}$, método helicoidal e colimação $2 \mathrm{~mm}$ ). $\mathrm{O}$ exame tomográfico de tórax não identificou alterações. E, para o exame de abdômen foram realizados cortes tomográficos de todo o abdômen com espessura de $5 \mathrm{~mm}$, o que indicou a presença de massa pedunculada hipoatenuante de lobo hepático médio direito, de contornos irregulares e textura grosseira e heterogênea, medindo $7,20 \times 6,50 \times$ $7,50 \mathrm{~cm}$ de diâmetro em eixo laterolateral, ventrodorsal e anteroposterior, respectivamente.

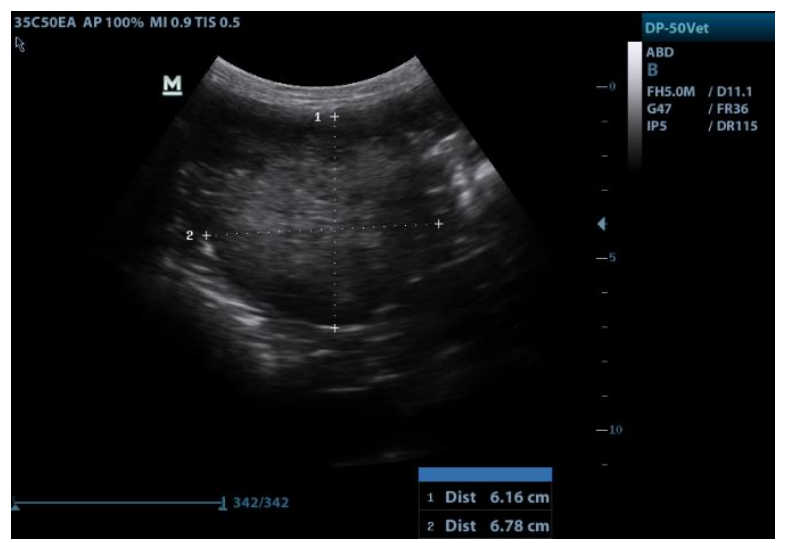

Figura 1. Imagem ultrassonográfica do fígado com observação e medição de estrutura amorfa de contornos irregulares e em região adjacente aos lobos hepáticos mediais. Observa-se a parênquima heterogêneo localizado sugestivo de neoplasma hepático focal.

O laudo tomográfico de abdômen foi sugestivo de neoplasma hepático, cuja indicação de tratamento inicial era a retirada da neoformação. Destaca-se que a paciente passou por avaliação cardiológica antes da cirurgia. Pouco dias depois, o animal foi submetido à hepatectomia parcial (lobectomia hepática) pela laparotomia medial. Devido ao quadro de hipoalbuminemia observado no hemograma e a possibilidade de hemorragia, optou-se por ter uma bolsa de sangue disponível durante $o$ ato cirúrgico.

Para a cirurgia a paciente recebeu como medicação pré-anestésica, midazolam $\left(0,3 \mathrm{mg} \cdot \mathrm{kg}^{-}\right.$ ${ }^{1}$ (IM)) associado ao tramadol (3 mg.kg-1 (IM)) para causar uma boa sedação e bom relaxamento muscular, tendo como vantagem relativa ausência de efeitos adversos em grupos de maior risco. (Fantoni \& Cortopassi, 2009). Realizou-se a tricotomia ampla do abdômen. No bloco cirúrgico, para a indução anestésica foi utilizado propofol na dose de $2 \mathrm{mg} \cdot \mathrm{kg}^{-1}$ (IV) e a manutenção do plano anestésico foi obtida com isoflurano por via inalatória (Fantoni \& Cortopassi, 2009). Destacase que a paciente recebeu antibiótico profilático, cefalotina na dose de $30 \mathrm{mg} \cdot \mathrm{kg}^{-1}$ (IV), 30 minutos antes do início da cirurgia (Fossum, 2014). Após todos os procedimentos pré-operatórios, a paciente foi posicionada em decúbito dorsal e foi 
realizada a antissepsia cirúrgica com álcool isopropílico $70 \%$ e iodopovidona a $10 \%$. A Figura $\underline{2}$ é possível observar o lobo acometido a ser extirpado por hepatectomia parcial.

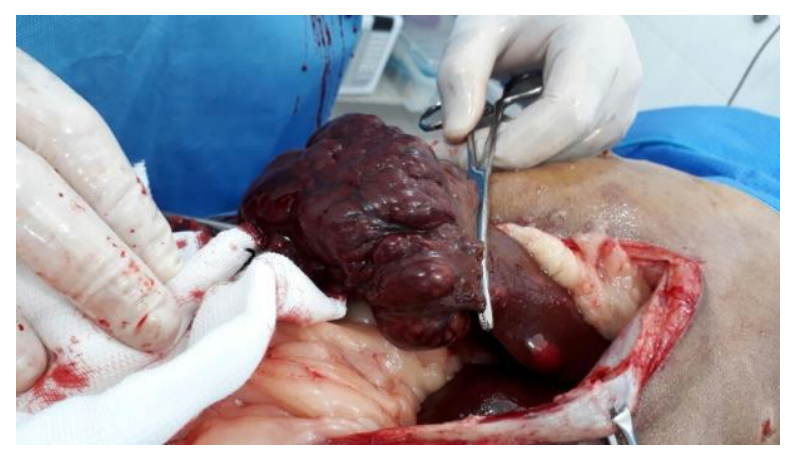

Figura 2. Aspecto macroscópico do lobo medial direito do fígado de um canino com hepatite crônica focal, mostrando múltiplos nódulos na superfície capsular hepática.

A paciente recuperou-se rapidamente após a cirurgia e, por não apresentar sinais de dor, recebeu alta no mesmo dia, com prescrição dos medicamentos: cefalexina por 10 dias, na dose de $30 \mathrm{mg} \cdot \mathrm{kg}^{-1}$ (VO) a cada $12 \mathrm{~h}$, ranitidina por 10 dias, na dose de $2 \mathrm{mg} \cdot \mathrm{kg}^{-1}(\mathrm{VO})$ a cada $12 \mathrm{~h}$, meloxicam por 3 dias, na dose de $0,1 \mathrm{mg} \cdot \mathrm{kg}^{-1}(\mathrm{VO})$ a cada $24 \mathrm{~h}$, tramadol por 5 dias na dose de $4 \mathrm{mg} \cdot \mathrm{kg}^{-1}$ (VO) a cada $8 \mathrm{~h}$, associado com dipirona na dose de 25 mg.kg-1 (VO) a cada 8h durante 7 dias (Spinosa et al., 1999; Fantoni \& Cortopassi, 2009; Papich, 2012). Foi indicada a limpeza diária da ferida cirúrgica com solução fisiológica três vezes ao dia, uso de colar Elisabetano, e, retorno em 10 dias para retirada dos pontos e reavaliação. Também foram prescritos: ácido ursodesoxicólico na dose de $15 \mathrm{mg} \cdot \mathrm{kg}^{-1}$ (VO) a cada $24 \mathrm{~h}$, s-adenosilmetionina na dose de $20 \mathrm{mg} . \mathrm{kg}-1$ (VO) a cada 24 $\mathrm{h}$ e vitamina E 400UI/animal a cada $24 \mathrm{~h}$, diariamente.

\section{Resultados e Discussão}

Nos cães, a doença hepática crônica é mais comum do que a doença aguda, e, geralmente leva a fibrose progressiva do órgão e cirrose. A causa da hepatite crônica em cães quase sempre é desconhecida, mas, o histórico de vacinação da paciente permite eliminar algumas causas, com: infecção por Adenovirus ou Leptospira. Ressaltase que no caso em pauta, o animal não apresentava qualquer sinal clínico de doença hepática, o que indica comprometimento da função hepática inferior a 75\% (Fossum, 2014), resultando em prognóstico favorável. Poldervaart et al. (2009) e

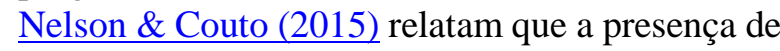

icterícia, ascite, hipoalbuminemia, desvio à esquerda no leucograma e linfonodos aumentados são associados a uma menor sobrevida em cães com hepatite primária. Portanto, o diagnóstico precoce das hepatopatias, antes do surgimento dos sinais clínicos, é muito benéfico para o tratamento ou controle da doença.

De acordo Fossum (2014), a lobectomia pode ser indicada para algumas lesões focais envolvendo um ou dois lobos hepáticos, contudo, a técnica deve ser realizada com cuidado, pois, o tecido hepático é friável, e, deve-se ter maior cuidado quando a massa está localizada em lobo direito do órgão. Para Vasconcellos (2013), na hepatectomia parcial de lobos direito e caudado, e, lobos esquerdos de cães maiores, é preciso dissecar cuidadosamente o lobo a partir da veia cava caudal, isolar os vasos sanguíneos e os ductos biliares próximos ao hilo e ligá-los duplamente, e, por fim, deve-se seccionar o tecido parenquimatoso, deixando um coto de tecido distalmente às ligaduras para evitar retração do tecido hepático e subsequente hemorragia.

Segundo Slatter (2007), após uma hepatectomia parcial, a regeneração do órgão tem início $24 \mathrm{~h}$ após o procedimento, em que o pico regenerativo ocorre em até 3 dias, devido a hipertrofia compensatória e hiperplasia dos hepatócitos remanescentes, restaurando em $70 \%$ a massa hepática por volta da sexta semana após a hepatectomia. $\mathrm{O}$ autor expõe que o estado geral do paciente e a saúde relativa da porção remanescente do fígado exercem grande efeito na sobrevida do animal.

O estudo histopatológico do lobo excisado resultou em hiperplasia hepatocelular com formação de pontes de fibrose, com presença de nódulos regenerativos contornados por material colagenizado, sendo alterações indicativas de hepatite crônica em resolução, evidenciando quadro de cirrose organizada. A Figura 3 apresenta a desorganização da arquitetura hepática, com áreas de fibrose, deposição de gordura e infiltração de células inflamatórias. Destaca-se que foram observadas outras alterações histopatológicas, como, hiperplasia de ducto biliar, congestão, colestase e telangiectasia, que são alterações secundárias à compressão e perda da arquitetura hepática decorrente da fibrose. Contudo, não foram observadas características de malignidade ou agentes infecciosos na amostra. 
Três meses após a hepatectomia o animal realizou outro exame ultrassonográfico para acompanhar sua evolução. Na figura 4 nota-se um fígado com dimensões moderadamente aumentadas, contornos regulares e focos hipogênicos dispersos no parênquima hepático. Os vasos hepáticos e portais estavam com calibres e arquitetura preservados.

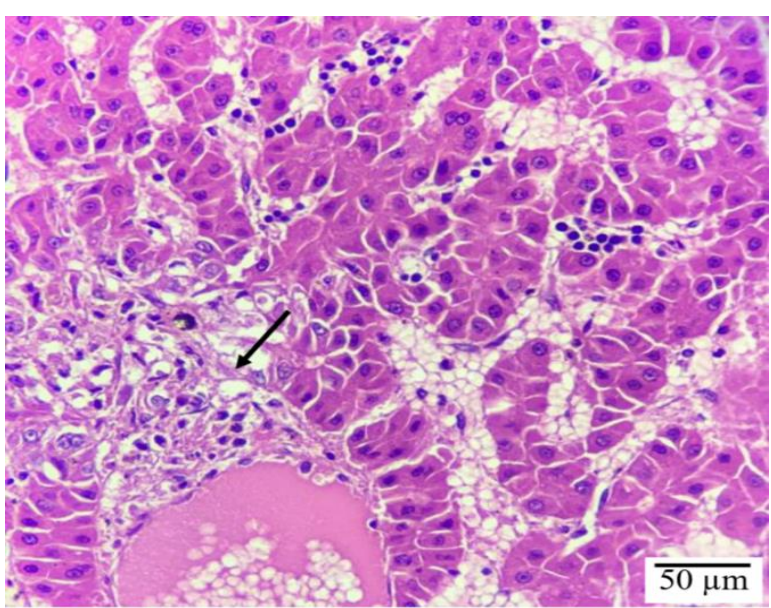

Figura 3. Presença de pontes de fibrina separando hepatócitos individualmente ou em pequenos grupos (seta).

Os tutores mantêm o monitoramento semestral da função hepática da paciente, cujo valor de ALT passou de 406 U.I./L, antes do tratamento, para uma média de 167 U.I./L. Já a fosfatase alcalina apresentou uma redução de, aproximadamente, 93\%, saindo de 2640 U.I./L para 185 U.I./L. Destaca-se que até o momento não foi observada nenhuma manifestação clínica ou comportamental que justificasse a alteração da dieta (ração super premium) para dieta uma dieta com baixo teor de proteína (ração terapêutica específica para hepatopatas).

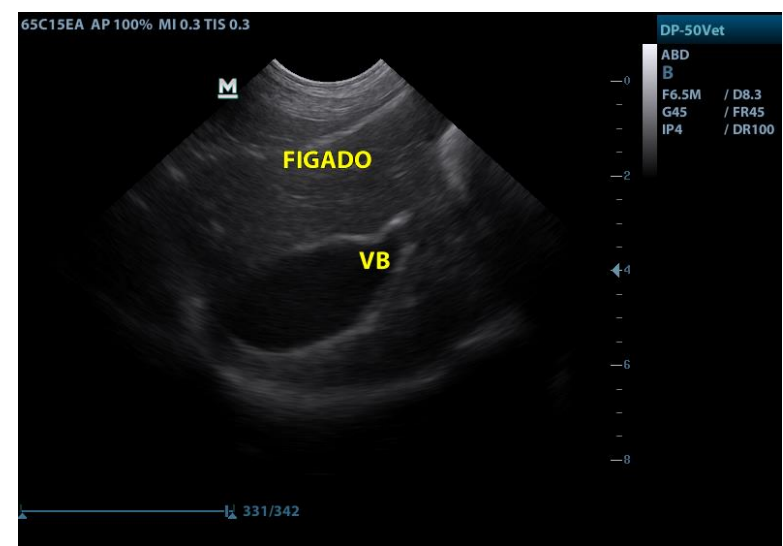

Figura 4. Imagem ultrassonográfica do fígado e vesícula bilar três meses após hepatectomia parcial para retirada do lobo medial direito.

\section{Conclusões}

A hepatite crônica focal, quando em lobo hepático único, apresenta prognóstico mais favorável do que em casos de hepatite crônica disseminada pelo órgão. A técnica de hepatectomia parcial se mostrou eficaz, apesar do maior grau de dificuldade cirúrgica devido a massa estar em lobo direto. A história clínica da paciente associada à conduta terapêutica adotada neste caso demonstra uma evolução positiva do quadro. Enfatiza-se que o sucesso no tratamento se deu, principalmente, pela precoce suspeita diagnóstica, permitindo boa evolução do caso. A continuidade do tratamento medicamentoso e o monitoramento periódico da função hepática demonstraram a eficácia da terapêutica instituída e os bons resultados na saúde do animal.

\section{Referências Bibliográficas}

Fantoni, D.T. \& Cortopassi, S.R.G., 2009. Anestesia em cães e gatos. Roca, São Paulo:, Brasil.

Fossum, T.W., 2014. Cirurgia de pequenos animais. Elsevier Brasil, São Paulo.

Nelson, R.W. \& Couto, C.G., 2015. Medicina interna de pequenos animais. Elsevier Editora, Amsterdan.

Papich, M.G., 2012. Manual Saunders de Terapia Veterinária. Elsevier Health Sciences Brazil.

Poldervaart, J.H., Favier, R.P., Penning, L.C. \& Van den Ingh, T.S.G.A.M., Rothuizen, J., 2009. Primary hepatitis in dogs: a retrospective review (2002-2006). Journal of Veterinary Internal Medicine 23, 72-80.

Richter, K.P., 2005. Doenças do fígado e do sistema hepatobiliar, In: Tams, T.R. (Ed.), Gastroenterologia de pequenos animais, Roca, São Pauylo, Brasil, pp. 283-348.

Schwarz, T., 2012. Fígado e Vesícula Biliar, In: O'Brien, R., Barr, F. (Eds.), Manual de Diagnóstico por Imagem Abdominal de Cães e Gatos, Roca, São Paulo, p. 188.

Silva, M.C., Fighera, R.A., Brum, J.S., Graça, D.L., Kommers, G.D., Irigoyen, L.F. \& Barros, C.S.L., 2007. Cirrose hepática em cães: 80 casos (1965-2003). Pesquisa Veterinária Brasileira 27, 471-480.

Slatter, D.H., 2007. Manual de cirurgia de pequenos animais. Manole, São Paulo.

Spinosa, H.S., Górniak, S.L. \& Bernardi, M.M., 1999. Farmacologia aplicada à medicina 
veterinária. Guanabara Koogan, Rio de Janeiro.

Tostes, R.A. \& Bandarra, E.P., 2004. Aspectos etiológicos, epidemiológicos e patológicos das hepatites crônicas em cães. Revista Científica de Medicina Veterinária 2, 67-72.

Vasconcellos, M., 2013. Aspectos cirúrgicos no tratamento de tumores hepatobiliares caninos: uma revisão. PUBVET,7, Art. 1609.

Webster, C.R.L., 2008. Cirrose e fibrose hepática, In: Tilley, L.P., Smith Junior., F.W.K. (Eds.),
Consulta veterinária em 5 minutos: espécies canina e felina, Manole, São Paulo, Brasil, pp. 226-228.

Recebido: 5 Jun., 2018.

Aprovado: 2 Jul., 2018

Publicado: 10 Ago., 2018

Licenciamento: Este artigo é publicado na modalidade Acesso Aberto sob a licença Creative Commons Atribuição 4.0 (CC-BY 4.0), a qual permite uso irrestrito, distribuição, reprodução em qualquer meio, desde que o autor e a fonte sejam devidamente creditados 